\title{
Effects of Hypercapnia in Lung Tissue Repair and Transplant
}

\author{
Stephen Chiu • Jacob Kanter • Haiying Sun • \\ Anchit Bharat • Peter H. S. Sporn • Ankit Bharat
}

Published online: 20 January 2015

(C) Springer International Publishing AG 2015

\begin{abstract}
Mammalian cells sense and transduce signals in response to high levels of carbon dioxide. Hypercapnia has a variety of effects on epithelial and immune cells which can be beneficial or detrimental depending on the biologic context. For instance, hypercapnia-mediated suppression of cell proliferation and migration can delay wound repair. Similarly, suppression of macrophages and the inflammatory response during hypercapnia can limit the host's ability to clear pathogens. However, the suppressive effects of hypercapnia on immunity have potential benefits in the setting of transplantation. Here, we discuss the effects of high levels of carbon dioxide on lung healing and the potential applications in lung transplantation.
\end{abstract}

Keywords Lung repair · Lung transplant $\cdot$ Hypercapnia . Carbon dioxide $\cdot$ Immunity $\cdot$ Cell migration $\cdot$ Pulmonary edema $\cdot$ Inflammation $\cdot$ Injury response $\cdot$ ARDS

This article is part of the Topical Collection on Tissue Engineering and Regeneration

S. Chiu $\cdot$ J. Kanter $\cdot$ H. Sun $\cdot$ A. Bharat

Division of Thoracic Surgery, Department of Surgery, Northwestern

University, Chicago, IL, USA

\section{A. Bharat $\cdot$ P. H. S. Sporn}

Division of Pulmonary \& Critical Care Medicine, Department of

Medicine, Northwestern University, Chicago, IL, USA

P. H. S. Sporn

Jesse Brown Veterans Affair Medical Center, Chicago, IL, USA

A. Bharat $(\bowtie)$

676 N Saint Clair St, Suite 650, Chicago, IL 60611, USA

e-mail: abharat@nm.org

\section{Introduction}

Carbon dioxide $(\mathrm{CO} 2)$ is a by-product of oxidative metabolism. The idea that $\mathrm{CO} 2$ regulates intracellular events is innovative and is currently being investigated in our laboratories and others $[1 \bullet 2 \bullet, 3-7]$. There is accumulating evidence indicating that mammalian cells sense and transduce signals in response to high levels of $\mathrm{CO} 2$. Although a variety of cells can sense and respond to $\mathrm{CO} 2$, the focus of this review is primarily on lung tissue repair and transplantation. Here, we will discuss our recently published data and hypotheses on the detrimental effects of high $\mathrm{CO} 2$ levels on the lung repair process. We will also present potential therapeutic benefits of elevated $\mathrm{CO} 2$ in lung transplantation.

\section{CO2 sensing}

$\mathrm{CO} 2$ is a byproduct of oxidative metabolism and is produced by the citric acid cycle within mitochondria. It is a small nonpolar molecule capable of passive diffusion across cell membranes. In some systems, especially those without a gradient, $\mathrm{CO} 2$ transport can be channel-mediated [8,9]. Once inside the cell, enzymes such as carbonic anhydrases can act as sensors of $\mathrm{CO} 2$ and mediate $\mathrm{CO} 2$ metabolism and signaling. In addition to sensing $\mathrm{CO} 2$, carbonic anhydrase can also facilitate its transport [10].

Another enzyme that mediates $\mathrm{CO} 2$ sensing is adenylyl cyclase (AC), responsible for the production of cyclic adenosine monophosphate (cAMP). Transmembrane adenylyl cyclases (TMACs) that are sensitive to G-proteins get activated secondary to ligand binding. Activation of TMAC leads to cAMP production that acts as a second messenger to induce effector proteins like protein kinase A, cAMP response element binding protein, phosphodiesterase domains, and cyclic nucleotide-gated ion channels. Elevated $\mathrm{CO} 2$ has been shown 
to suppress cAMP production and can, thereby, affect a variety of intracellular processes. There is evidence for direct incorporation of $\mathrm{CO} 2$ into a $\mathrm{CO} 2$-sensitive TMAC, implying that there is a direct pathway for $\mathrm{CO} 2$ sensing [11]. Additionally, $\mathrm{CO} 2$ can also affect soluble adenylyl cyclases.

In mammalian cells, regulation of $\mathrm{CO} 2$ is important since it affects ventilation, alveolar function, cell proliferation, inflammation, and innate immune responses. Neurons located in the brainstem detect changes in arterial pCO2 and in turn regulate breathing $[12,13]$. Central and peripheral chemoreceptors modulate each other so that the carotid body can fine tune the sensitivity of central chemoreceptors under hypercapnia [14]. An increase in blood arterial CO2 can lead to depolarization of glomus cells, triggering an increase in alveolar ventilation to maintain normal pCO2 [15]. $\mathrm{CO} 2$ also regulates a variety of other cellular responses, including those of the respiratory tract.

\section{$\mathrm{CO} 2$ and the respiratory tract}

The lung epithelium is an interface with the environment and can be subject to many injury mechanisms. This interface is composed of alveolar epithelium consisting of type I and type II pneumocytes and the endothelium of pulmonary capillaries. Damage to the alveoli has the biggest effect on respiratory physiology. During normal homeostasis, lung cells exhibit a low rate of cell regeneration. After injury, they have the capacity to rapidly regenerate through activation of progenitor cells and proliferation of remaining uninjured cells. Lung repair results in either complete resolution of injury, persistent chronic inflammation, remodeling with fibrosis, or progressive ongoing fibrosis. Without complete resolution, permanent reduction in lung function occurs.

While an increase in blood $\mathrm{pCO} 2$ is known to trigger an increase in respiration by central regulation [16], there is growing evidence to support $\mathrm{CO} 2$ sensing and regulation of intracellular events in the epithelial and immune cells of the respiratory tract. The effects of $\mathrm{CO} 2$ are particularly important in the context of lung injury. Severe injury to the lungs results in the disruption of the alveolar-capillary barrier. Such an impact is a hallmark of acute respiratory distress syndrome (ARDS) and leads to impaired gas exchange. After resolution of the inciting event, the lung undergoes repair. Although lung repair is a highly complex process, there are some key components including the following: A) Clearance of cellular debris by phagocytic cells; and B) Proliferation and migration of alveolar type II cells to cover the injured area. Once the injury response has occurred, removal of edema fluid is necessary for lung function to recover. Impairment of this process leads to pulmonary fibrosis and to a decline in lung function.
Effects of $\mathrm{CO} 2$ on lung macrophages

Macrophages are the most well-established scavengers and, therefore, important in the wound healing process. There is now strong evidence to support that hypercapnia can alter macrophage function. This includes both regulation of the phagocyte function as well as cytokine/chemokine production. West and colleagues investigated why laparoscopic surgery that utilizes $\mathrm{CO} 2$ was associated with decreased peritoneal inflammation [17]. They found that peritoneal macrophages did not produce tumor necrosis factor (TNF) and interleukin (IL)-1beta upon lipopolysaccharide (LPS) stimulation under hypercapnia. A similar observation was made in rat alveolar macrophages [18]. Investigation performed on LPSstimulated human and mouse macrophages revealed that elevated $\mathrm{CO} 2$ reduced the expression of TNF and IL-6 [5]. In the above studies, hypercapnia affected NF-kB-regulated cytokines but not those that are not regulated by this pathway. The NF- $\mathrm{kB}$ pathway plays a key role in the regulation of innate immunity and inflammation [19]. Hence, this pathway may be important in hypercapnia-mediated gene regulation. As such, the suppression of these cytokines by hypercapnia occurs at the gene level and not due to cytotoxicity. Takeshita and colleagues showed that hypercapnia inhibited endotoxinstimulated RelA nuclear translocation that is an important component of NF- $\mathrm{KB}$ signaling [20]. Hypercapnia might also affect the non-canonical NF- $\mathrm{KB}$ pathway through the regulation of IKK $\alpha$ and RelB signaling [21•, 22, 23]. Furthermore, elevated $\mathrm{CO} 2$ can alter pulmonary inflammation in an NF- $\mathrm{KB}$ -dependent manner [24].

Macrophages are known to phagocytose pathogens. In addition, through the process of efferocytosis, they clear cellular debris. Besides altering innate immune gene expression, hypercapnia also inhibits phagocytosis [5] and NADPH oxidase-mediated generation of reactive oxygen species [2•], which is necessary for antimicrobial activity. Hypercapnia was found to worsen lung injury in rats with Escherichia coli pneumonia [25]. Further, hypercapnia markedly increases the mortality of $P$. aeruginosa pneumonia in a rodent model by decreasing the clearance of Pseudomonas from the lungs and increasing bacterial dissemination to other organs [2•]. The hypercapnia-induced increase in pneumonia mortality was not due to acidosis because mortality in mice pre-exposed to hypercapnia for three days, to allow for metabolic compensation, was similar to those that were not pre-exposed. In our ongoing studies, we have further found that the efferocytosis of apoptotic alveolar epithelial cells by alveolar macrophages is inhibited in hypercapnia. This leads to impairment of wound healing due to persistence of apoptotic cells at the injury site. Although both the engulfment of the apoptotic cells and digestion of phagosomes are affected in hypercapnia, the latter is more pronounced. Transfer of macrophages that have engulfed apoptoti cells in normocapnic conditions to 
hypercapnia significantly slows phagosome digestion. It appears that this might be mediated by suppression of Rab5 GTPase that is important for phagosome-lysosome fusion (manuscript under preparation). Such a delay in clearance of cellular debris can results in poor healing following lung injury. In fact, there is now evidence to support that defective macrophage function and reduced ability to clear apoptotic cells can lead to progressive pulmonary fibrosis both in native lungs [26] and transplanted lung allografts [27].

Effects of $\mathrm{CO} 2$ on lung epithelium

The disruption of the plasma membrane is an important component of a variety of injury mechanisms including ARDS $[28,29]$. Hypercapnia can have a profound effect on the lung injury response. Most cells under a normocapnic environment repair even micron-scale defects in the plasma membranes within 30 seconds [30]. However, under hypercapnia, the recovery is significantly delayed, resulting in cell death. $\mathrm{CO} 2$ also has direct intracellular effects on lung epithelial cells. Alveolar type II cells are key mediators of alveolar injury response. They proliferate in response to the injury and are responsible for differentiating into type I cells as well as closing the injury defect. In a previously published study, hypercapnia was found to induce mitochondrial dysfunction in alveolar type II cells, suppressing cell proliferation and wound closure [31]. This was a result of suppression of isocitrate dehydrogenase-2 (IDH-2), a citric acid cycle enzyme. Further, suppression of IDH-2 was mediated by hypercapnia-induced upregulation of microRNA 183 (miR-183). MicroRNAs belong to the family of short post-transcriptional inhibitor RNAs. Transfection with IDH-2 reversed the delayed wound closure under hypercapnia. Interestingly, treatment with antimiR-183 (antagomir) reverses the suppression of cell proliferation under hypercapnia but nota the delayed wound closure (manuscript under preparation). This indicates that hypercapnia suppresses cell proliferation and migration through an independent pathway. Studies are underway to elucidate the mechanisms that lead to impairment of cell migration in our laboratories.

Effects of $\mathrm{CO} 2$ on pulmonary edema clearance

Clearance of pulmonary edema is dependent on the sodium channel $\mathrm{Na}, \mathrm{K}$-ATPases located on the alveolar epithelium [32, 33]. Clearance of the excess alveolar fluid is essential for recovery following lung injury. It is noteworthy that ventilator management for patients with ARDS includes protective lung ventilation using low tidal volume ventilation that invariably leads to hypercapnia. The hypercapnia is considered "permissive" in order to allow the protective lung ventilation. However, high $\mathrm{CO} 2$ is not without consequences. Hypercapnia impairs Na,K-ATPase function and alveolar edema clearance
[34]. Hypercapnia led to activation of PKC- $\zeta$, which directly phosphorylates the Na,K-ATPase $\alpha 1$-subunit at the Ser 18 residue, leading to endocytosis of this pump and decreased alveolar fluid clearance. The activation of $\mathrm{PKC} \zeta$ is dependent on the JNK-required AMP-activated protein kinase (AMPK) $[34,35]$. Hence, down-regulation of Na,K-ATPase in hypercapnia is distinct from other stimuli that mediate the same effect by the production of cytosolic and mitochondrial reactive oxygen species [35-38]. Further, these effects are independent of extracellular and intracellular acidosis. Cumulatively, hypercapnia results in epithelial cell dysfunction.

\section{$\mathrm{CO} 2$ in ARDS and pneumonia}

ARDS is characterized by severe damage to the alveolarcapillary membranes. Protein-rich fluid seeps into the alveoli and lead to interstitial edema. This leads to characteristic decreased lung compliance and diffuse bilateral infiltrates on chest radiographs. The loss of this mechanical barrier predisposes patients to bacterial invasion and sepsis. There is also loss of fluid and ion transport, exposure of the immunogenic basement membrane, and activation of neutrophils and macrophages. The management of ARDS typically includes protective lung ventilation using low tidal volume and airway pressure ventilation. This mechanical ventilation strategy was first described for ARDS in the 1990s and has since been proven to be associated with lower hospital mortality. Low tidal volumes are thought to reduce atelectotrauma and break the cycle of recruitment and collapse of atelectatic lung. However, low tidal volume also leads to hypercapnia and acidosis. Over time, the acidosis gets corrected but hypercapnia usually persists as long as the patient is on low tidal volume ventilation. A current area of controversy is whether or not hypercapnia itself is beneficial in the treatment of ARDS [39-43]. Proponents of hypercapnia argue that it attenuates oxygen free radical formation, NF- $\mathrm{KB}$ activation, and TNF- $\alpha$, IL-1, IL-6, and IL-8 cytokine production $[1 \bullet, 22]$. Indeed, hypercapnia, has been shown to attenuate lung injury in animal models of barotrauma $[44,45]$ and by endotoxin in rats [46]. However, opponents argue that hypercapnia delays bacterial clearance and worsens lung injury in long standing pneumonia and sepsis [25]. Furthermore, hypercapnia causes mitochondrial dysfunction and impairs alveolar cell proliferation [31]. We favor that hypercapnia is detrimental to lung repair and recovery during pneumonia and ARDS. This is further supported by the fact that hypercapnia is an independent risk factor for mortality in patients with community-acquired pneumonia and multiple adverse clinical outcomes, including pneumonia, sepsis, and mortality [47, 48]. 


\section{$\mathrm{CO} 2$ and lung transplantation}

Lung transplants are increasingly being used as a treatment option for a variety of lung disorders, including emphysema, cystic fibrosis, pulmonary fibrosis, and pulmonary vascular diseases, among others. Although there has been significant improvement in the surgical technique and immunosuppression protocols, the long-term outcomes are still limited due to the development of chronic allograft rejection, called bronchiolitis obliterans syndrome (BOS). It is estimated that up to $50 \%$ of lung transplant recipients will develop BOS after 5 years and the median survival rate following BOS is only 2-3 years $[49,50]$. Hence, BOS currently remains the bane of lung transplants. Unfortunately, the pathogenesis of BOS is still undefined, although a variety of injury mechanisms have been postulated [51]. These include primary graft dysfunction (PGD), acute cellular rejection, humoral rejection, respiratory viral infections, gastroesophageal reflux, and autoimmunity. Recent studies have demonstrated that PGD is one of the most important risk factors for BOS $[52,53]$. PGD occurs within the first 72 hours following lung transplantation and can affect over $25 \%$ of lung transplant recipients. Ischemia reperfusion injury is the main contributor to the development of PGD and there is an emerging role of innate immunity. The suppressive effects of hypercapnia on innate immunity might therefore suggest a potential therapeutic role in ameliorating ischemia reperfusion injury. Li et al demonstrated that suppression of NF-KB activation lead to a reduction in rat hepatic ischemia reperfusion injury under hypercapnia [54]. Similar observations have been made in lung [55] and gut ischemia-reperfusion models [56]. Hence, transient exposure to hypercapnia might be beneficial in reducing ischemia reperfusion injury.

A number of immune pathways have been implicated in the development of PGD. Kohno and colleagues demonstrated that Rho GTPase and Rho-associated coiledcoil forming protein kinase (ROCK) regulate the contraction of endothelial cells by modulating the intracellular cytoskeleton via phosphorylation of the endothelial

Table 1 Hypercapnia and Lung Healing

\begin{tabular}{lc}
\hline Inflammatory response inhibition & Repair/regeneration inhibition \\
\hline NADPH oxidase generation of & \\
O2 radicals & \\
Macrophage production of TNF & Alveolar edema clearance via \\
alpha, IL-1, IL-6, IL-8 & Na,K-ATPase inhibition \\
NF-kappaB mediated cytokine & Cell proliferation via inhibition \\
release & of Krebs cycle \\
Macrophage phagocytosis & Cell migration via inhibition \\
& of Rac1-GTPase \\
\hline
\end{tabular}

Table 2 Hypercapnia and Lung Transplant

\begin{tabular}{ll}
\hline Therapeutic potential & Negative potential \\
\hline $\begin{array}{l}\text { Decreased ischemia-reperfusion } \\
\text { injury }\end{array}$ & Increased risk of infection \\
$\begin{array}{l}\text { Suppression of donor-derived } \\
\text { macrophages }\end{array}$ & $\begin{array}{c}\text { Decreased pathogen-specific } \\
\text { immunity }\end{array}$ \\
Suppression of endothelial & Pulmonary artery \\
Rho-GTPase & vasoconstriction \\
Suppression of NF-kappaB activation & Pulmonary artery hypertension \\
\hline
\end{tabular}

myosin light chain. Contraction of the endothelial cells allows for transendothelial cell migration and increases permeability, contributing to reperfusion injury. Inhibition of this pathway by the addition of Y-27632, a specific ROCK inhibitor, to the preservative solution during the cold ischemia significantly ameliorated the ischemia reperfusion injury $[57,58]$. Peng and colleagues demonstrated that inhibition of ROCK can be achieved to the same degree by $10 \% \mathrm{CO} 2$ [59]. Hence, hypercapnia might be protective against ischemia-reperfusion injury.

Several prior studies have demonstrated that infiltration of recipient-derived macrophages, neutrophils, NK cells, and T cells are important for immunity against pathogens contained in the donor lung [60]. However, they also mediate ischemia reperfusion injury and PGD following transplantation. Inflammatory cytokines produced as a result of the cellular infiltration are important for the development of PGD. Furthermore, the inflammatory milieu triggers development of alloimmunity against donor HLA antigens as well as autoimmunity against lung tissuerestricted antigens. Sharma and colleagues reported that IL-17 and TNF- $\alpha$ from NK T cells and alveolar macrophages contribute significantly to ischemia reperfusion injury and PGD. Both these cytokines synergistically mediate CXCL1 production (neutrophil chemoattractant) by alveolar type II epithelial cells via a NADPH oxidasedependent mechanism [61]. In fact, NADPH oxidase is important for all bone marrow-derived immune cells that contribute to ischemia reperfusion injury in lung transplant recipients [62] which is also suppressed by hypercapnia [2•]. Hence, there is a potential benefit of using hypercapnia in lung transplantation to reduce ischemia reperfusion injury. The caveat remains that hypercapnia can also lead to suppression of pathogen-specific immunity. Infections remain the predominant cause of morbidity and mortality during the early post-transplant period. Hence, the potential immunomodulatory benefits of hypercapnia should be weighed against increased infection risk. Nevertheless, we postulate that transient hypercapnia in the immediate post-transplant period would have a more favorable impact in PGD while not significantly increasing the infection risk. If used in the context of 
lung transplantation, the timing of hypercapnia will be important. Hypercapnia increases pulmonary arterial pressures. Clamping the pulmonary artery (right or left) for the implantation will further raise the pulmonary arterial pressures making it difficult to perform the transplantation without the need for cardiopulmonary bypass. Hence, it might be difficult to use this strategy intraoperatively. However, most lung transplant recipients remain intubated for a period of time after transplantation. Following completion of the lung transplant, the pulmonary pressures are usually low and permissive hypercapnia would be well tolerated. Another possibility is inducing hypercapnia during cold storage. The role of donorderived macrophages in ischemia reperfusion injury remains unclear. Nevertheless, it has been postulated that elimination or suppression of donor lung macrophages reduces ischemia reperfusion injury. Therefore, hypercapnia-mediated suppression of these macrophages prior to reperfusion is potentially beneficial. Furthermore, suppression of Rho-GTPase in the endothelial cells by hypercapnia during the cold ischemia could also reduce capillary permeability and cellular infiltration in the allograft following reperfusion. The development of the ex vivo lung perfusion system [63] presents an exciting opportunity to test some of these hypotheses.

\section{Conclusions}

The effects of hypercapnia on mammalian cells are being defined. Depending on the clinical situation, hypercapnia can be detrimental or beneficial (Table 1). When the suppression of the immune response is important, for example, in ischemia-reperfusion injury in organ transplantations, it can be utilized for therapeutic benefit (Table 2). However, when the immune response is important, for example in the setting of pneumonia, it can be detrimental. In the setting of lung repair, although it has benefits in reducing the inflammatory response, the overall effects are detrimental due to the reasons outlined above. Ongoing investigations are important to delineate the effects of hypercapnia in lung repair, transplantation, and perhaps regeneration.

\section{Compliance with Ethics Guidelines}

Conflict of Interest Stephen Chiu, Jacob Kanter, Haiying Sun, Anchit Bharat, Peter H. S. Sporn, and Ankit Bharat declare that they have no conflict of interest.

Human and Animal Rights and Informed Consent This article does not contain any studies with human or animal subjects performed by any of the authors.

\section{References}

Papers of particular interest, published recently, have been highlighted as:

- Of importance

1. Cummins EP et al. Carbon dioxide-sensing in organisms and its implications for human disease. Cell Mol Life Sci. 2014;71(5): 831-45. This article is a comprehensive review of the mechanisms of $\mathrm{CO} 2$ sensing in eukaryotic and mamalian cells.

2. Gates KL et al. Hypercapnia impairs lung neutrophil function and increases mortality in murine pseudomonas pneumonia. Am J Respir Cell Mol Biol. 2013;49(5):821-8. In this article, Gates and colleagues demonstate impairment of pathogen-specific immunity under hypercapnic conditions.

3. Do-Umehara HC et al. Suppression of inflammation and acute lung injury by Miz1 via repression of C/EBP-delta. Nat Immunol. 2013;14(5):461-9.

4. Vadasz I et al. Hypercapnia: a nonpermissive environment for the lung. Am J Respir Cell Mol Biol. 2012;46(4):417-21.

5. Wang $\mathrm{N}$ et al. Elevated $\mathrm{CO} 2$ selectively inhibits interleukin- 6 and tumor necrosis factor expression and decreases phagocytosis in the macrophage. FASEB J. 2010;24(7):2178-90.

6. Helenius IT et al. Elevated CO2 suppresses specific Drosophila innate immune responses and resistance to bacterial infection. Proc Natl Acad Sci U S A. 2009;106(44):18710-5.

7. Sloniewsky DE et al. Leukotriene D4 activates alveolar epithelial $\mathrm{Na}, \mathrm{K}-\mathrm{ATP}$ ase and increases alveolar fluid clearance. Am J Respir Crit Care Med. 2004;169(3):407-12.

8. Perry SF et al. Do zebrafish Rh proteins act as dual ammonia-CO2 channels? J Exp Zool A Ecol Genet Physiol. 2010;313(9):618-21.

9. Missner A et al. Carbon dioxide transport through membranes. J Biol Chem. 2008;283(37):25340-7.

10. Xue $\mathrm{S}$ et al. Central functions of bicarbonate in S-type anion channel activation and OST1 protein kinase in $\mathrm{CO} 2$ signal transduction in guard cell. EMBO J. 2011;30(8):1645-58.

11. Townsend PD et al. Stimulation of mammalian G-proteinresponsive adenylyl cyclases by carbon dioxide. J Biol Chem. 2009;284(2):784-91.

12. Huckstepp RT, Dale N. Redefining the components of central CO2 chemosensitivity-towards a better understanding of mechanism. J Physiol. 2011;589(Pt 23):5561-79.

13. Forster $\mathrm{HV}$ et al. The carotid chemoreceptors are a major determinant of ventilatory $\mathrm{CO} 2$ sensitivity and of $\mathrm{PaCO} 2$ during eupneic breathing. Adv Exp Med Biol. 2008;605:322-6.

14. Blain GM et al. Peripheral chemoreceptors determine the respiratory sensitivity of central chemoreceptors to $\mathrm{CO}(2)$. J Physiol. 2010;588(Pt 13):2455-71.

15. Musa-Aziz R et al. Relative $\mathrm{CO} 2 / \mathrm{NH} 3$ selectivities of $\mathrm{AQP} 1$, AQP4, AQP5, AmtB, and RhAG. Proc Natl Acad Sci U S A. 2009;106(13):5406-11.

16. Putnam RW, Filosa JA, Ritucci NA. Cellular mechanisms involved in $\mathrm{CO}(2)$ and acid signaling in chemosensitive neurons. Am J Physiol Cell Physiol. 2004;287(6):C1493-526.

17. West MA et al. Mechanism of decreased in vitro murine macrophage cytokine release after exposure to carbon dioxide: relevance to laparoscopic surgery. Ann Surg. 1997;226(2):17990.

18. Lang CJ et al. Effect of CO2 on LPS-induced cytokine responses in rat alveolar macrophages. Am J Physiol Lung Cell Mol Physiol. 2005;289(1):L96-103. 
19. Hayden MS, Ghosh S. NF-kappaB, the first quarter-century: remarkable progress and outstanding questions. Genes Dev. 2012;26(3):203-34.

20. Takeshita $\mathrm{K}$ et al. Hypercapnic acidosis attenuates endotoxininduced nuclear factor-[kappa]B activation. Am J Respir Cell Mol Biol. 2003;29(1):124-32.

21. Oliver KM et al. Hypercapnia induces cleavage and nuclear localization of RelB protein, giving insight into $\mathrm{CO} 2$ sensing and signaling. J Biol Chem. 2012;287(17):14004-11. Oliver and colleagues show evidence that CO2 can affect the NF-kappaB pathway and, therefore, might have broad implications in humans.

22. Cummins EP et al. NF-kappaB links CO2 sensing to innate immunity and inflammation in mammalian cells. J Immunol. 2010;185(7):4439-45.

23. Taylor CT, Cummins EP. Regulation of gene expression by carbon dioxide. J Physiol. 2011;589(Pt 4):797-803.

24. Abolhassani $\mathrm{M}$ et al. Carbon dioxide inhalation causes pulmonary inflammation. Am J Physiol Lung Cell Mol Physiol. 2009;296(4): L657-65.

25. O'Croinin DF et al. Sustained hypercapnic acidosis during pulmonary infection increases bacterial load and worsens lung injury. Crit Care Med. 2008;36(7):2128-35.

26. Morimoto K, Janssen WJ, Terada M. Defective efferocytosis by alveolar macrophages in IPF patients. Respir Med. 2012;106(12): 1800-3.

27. Hodge $\mathrm{S}$ et al. Decreased efferocytosis and mannose binding lectin in the airway in bronchiolitis obliterans syndrome. J Heart Lung Transplant. 2011;30(5):589-95.

28. Vlahakis NE, Hubmayr RD, Invited review: plasma membrane stress failure in alveolar epithelial cells. J Appl Physiol (1985), 2000. 89(6): p. 2490-6; discussion 2497.

29. Dreyfuss D et al. Intermittent positive-pressure hyperventilation with high inflation pressures produces pulmonary microvascular injury in rats. Am Rev Respir Dis. 1985;132(4):880-4.

30. Godin LM et al. Spatiotemporal dynamics of actin remodeling and endomembrane trafficking in alveolar epithelial type I cell wound healing. Am J Physiol Lung Cell Mol Physiol. 2011;300(4):L615-23.

31. Vohwinkel $\mathrm{CU}$ et al. Elevated $\mathrm{CO}(2)$ levels cause mitochondrial dysfunction and impair cell proliferation. J Biol Chem. 2011;286(43):37067-76.

32. Matthay MA, Folkesson HG, Clerici C. Lung epithelial fluid transport and the resolution of pulmonary edema. Physiol Rev. 2002;82(3):569-600.

33. Vadasz I, Raviv S, Sznajder JI. Alveolar epithelium and Na, KATPase in acute lung injury. Intensive Care Med. 2007;33(7): 1243-51.

34. Briva $\mathrm{A}$ et al. High $\mathrm{CO} 2$ levels impair alveolar epithelial function independently of pH. PLoS One. 2007;2(11):e1238.

35. Vadasz I et al. AMP-activated protein kinase regulates $\mathrm{CO} 2$-induced alveolar epithelial dysfunction in rats and human cells by promoting $\mathrm{Na}, \mathrm{K}-\mathrm{ATP}$ ase endocytosis. J Clin Invest. 2008;118(2):752-62.

36. Bertorello AM, Sznajder JI. The dopamine paradox in lung and kidney epithelia: sharing the same target but operating different signaling networks. Am J Respir Cell Mol Biol. 2005;33(5):432-7.

37. Dada LA et al. Hypoxia-induced endocytosis of $\mathrm{Na}$, K-ATPase in alveolar epithelial cells is mediated by mitochondrial reactive oxygen species and PKC-zeta. J Clin Invest. 2003;111(7):1057-64

38. Chen $\mathrm{J}$ et al. Carbonic anhydrase II and alveolar fluid reabsorption during hypercapnia. Am J Respir Cell Mol Biol. 2008;38(1):32-7.

39. Amato MB et al. Effect of a protective-ventilation strategy on mortality in the acute respiratory distress syndrome. N Engl J Med. 1998;338(6):347-54.

40. Laffey JG, Kavanagh BP. Ventilation with lower tidal volumes as compared with traditional tidal volumes for acute lung injury. $\mathrm{N}$ Engl J Med. 2000;343(11):812. author reply 813-4.
41. Laffey JG, Kavanagh BP. Ventilation with lower tidal volumes as compared with traditional tidal volumes for acute lung injury and the acute respiratory distress syndrome. The Acute Respiratory Distress Syndrome Network. N Eng1 J Med. 2000;342(18):1301-8.

42. Laffey JG, Kavanagh BP. Carbon dioxide and the critically ill-too little of a good thing? Lancet. 1999;354(9186):1283-6.

43. Curley GF, Laffey JG, Kavanagh BP. CrossTalk proposal: there is added benefit to providing permissive hypercapnia in the treatment of ARDS. J Physiol. 2013;591(Pt 11):2763-5.

44. Broccard AF et al. Protective effects of hypercapnic acidosis on ventilator-induced lung injury. Am J Respir Crit Care Med. 2001;164(5):802-6.

45. Sinclair SE et al. Hypercapnic acidosis is protective in an in vivo model of ventilator-induced lung injury. Am J Respir Crit Care Med. 2002;166(3):403-8.

46. Laffey JG et al. Hypercapnic acidosis attenuates endotoxin-induced acute lung injury. Am J Respir Crit Care Med. 2004;169(1):46-56.

47. Sin DD, Man SF, Marrie TJ. Arterial carbon dioxide tension on admission as a marker of in-hospital mortality in communityacquired pneumonia. Am J Med. 2005;118(2):145-50.

48. Laserna E et al. Hypocapnia and hypercapnia are predictors for ICU admission and mortality in hospitalized patients with communityacquired pneumonia. Chest. 2012;142(5):1193-9.

49. Trulock EP et al. Registry of the International Society for Heart and Lung Transplantation: twenty-second official adult lung and heartlung transplant report-2005. J Heart Lung Transplant. 2005;24(8): 956-67.

50. Valentine VG et al. Total lymphoid irradiation for refractory acute rejection in heart-lung and lung allografts. Chest. 1996;109(5):1184-9.

51. Bharat A, Mohanakumar T. Autoimmunity and lung transplantation. Front Biosci (Elite Ed). 2012;4:2378-88.

52. Bharat A et al. Immunological link between primary graft dysfunction and chronic lung allograft rejection. Ann Thorac Surg. 2008;86(1):189-95. discussion 196-7.

53. Hachem RR. Lung allograft rejection: diagnosis and management. Curr Opin Organ Transplant. 2009;14(5):477-82.

54. Li AM et al. Effects of therapeutic hypercapnia on inflammation and apoptosis after hepatic ischemia-reperfusion injury in rats. Chin Med J (Engl). 2010;123(16):2254-8.

55. Laffey JG et al. Therapeutic hypercapnia reduces pulmonary and systemic injury following in vivo lung reperfusion. Am J Respir Crit Care Med. 2000;162(6):2287-94.

56. Laffey JG et al. Effects of therapeutic hypercapnia on mesenteric ischemia-reperfusion injury. Am J Respir Crit Care Med. 2003;168(11):1383-90.

57. Tasaka $\mathrm{S}$ et al. Attenuation of endotoxin-induced acute lung injury by the Rho-associated kinase inhibitor, Y-27632. Am J Respir Cell Mol Biol. 2005;32(6):504-10.

58. Sawafuji $\mathrm{M}$ et al. Role of Rho-kinase in reexpansion pulmonary edema in rabbits. Am J Physiol Lung Cell Mol Physiol. 2005;289(6):L946-53.

59. Peng $\mathrm{G}$ et al. Sustained therapeutic hypercapnia attenuates pulmonary arterial Rho-kinase activity and ameliorates chronic hypoxic pulmonary hypertension in juvenile rats. Am J Physiol Heart Circ Physiol. 2012;302(12):H2599-611.

60. Todd JL, Palmer SM. Bronchiolitis obliterans syndrome: the final frontier for lung transplantation. Chest. 2011;140(2):502-8.

61. Sharma AK et al. NADPH oxidase mediates synergistic effects of IL-17 and TNF-alpha on CXCL1 expression by epithelial cells after lung ischemia-reperfusion. Am J Physiol Lung Cell Mol Physiol. 2014;306(1):L69-79.

62. Yang $\mathrm{Z}$ et al. NADPH oxidase in bone marrow-derived cells mediates pulmonary ischemia-reperfusion injury. Am J Respir Cell Mol Biol. 2009;40(3):375-81.

63. Cypel $\mathrm{M}$ et al. Normothermic ex vivo lung perfusion in clinical lung transplantation. N Engl J Med. 2011;364(15):1431-40. 